\title{
Arsenic in ZnO and GaN: Substitutional Cation or Anion Sites?
}

Ulrich Wahl ${ }^{1,2}$, Joao Guilherme Correia ${ }^{1,2}$, Elisabete Rita ${ }^{2}$, Ana Claudia Marques ${ }^{2,3}$, Eduardo Alves ${ }^{1,2}$, Jose Carvalho Soares ${ }^{2}$, and The ISOLDE Collaboration ${ }^{3}$

${ }^{1}$ Dep. Física, Instituto Tecnologico e Nuclear, Estrada Nacional 10, 2686-953 Sacavem, Portugal

${ }^{2}$ Centro de Fisica Nuclear da Universidade de Lisboa, Av. Prof. Gama Pinto 2, 1649-003 Lisbon, Portugal

${ }^{3}$ CERN, Geneva 23, 1211, Switzerland

\begin{abstract}
We have determined the lattice location of ion implanted As in $\mathrm{ZnO}$ and $\mathrm{GaN}$ by means of conversion electron emission channeling from radioactive ${ }^{73}$ As. In contrast to what one might expect from its nature as a group $\mathrm{V}$ element, we find that As does not occupy substitutional $\mathrm{O}$ sites in $\mathrm{ZnO}$ but in its large majority substitutional $\mathrm{Zn}$ sites. Arsenic in $\mathrm{ZnO}$ is thus an interesting example for an impurity in a semiconductor where the major impurity lattice site is determined by atomic size and electronegativity rather than its position in the periodic system. In contrast, in GaN the preference of As for substitutional cation sites is less pronounced and about half of the implanted As atoms occupy Ga and the other half $\mathrm{N}$ sites. Apparently, the smaller size-mismatch between As and $\mathrm{N}$ and the chemical similarity of both elements make it feasible that As partly substitutes for $\mathrm{N}$ atoms.
\end{abstract}

\section{INTRODUCTION}

Modifying the properties of $\mathrm{ZnO}$ and $\mathrm{GaN}$ by means of incorporating group $\mathrm{V}$ impurities is of interest in both of these wide band gap semiconductors, although for different reasons. In the case of the technologically promising II-VI compound $\mathrm{ZnO}$, besides $\mathrm{N}[1,2]$ the heavy group- $\mathrm{V}$ elements P [1,2], As [2-9], and Sb [10-12] have been reported in the literature as possible $p$-type dopants. However, there is an ongoing debate whether for $\mathrm{P}, \mathrm{As}$, and $\mathrm{Sb}$ the $p$-type character results from these impuritities simply replacing $\mathrm{O}$ atoms, thus acting as simple "chemical" dopants $[3,4,8]$, or is due to the formation of more complicated defect complexes [9,12-14]. The relative sizes of $\mathrm{Zn}, \mathrm{O}$ and $\mathrm{P}$, As, or $\mathrm{Sb}$ atoms favour incorporation of the heavy group- $\mathrm{V}$ elements on cation sites. Theoretical calculations have suggested that the acceptor action of arsenic in $\mathrm{ZnO}$ results from its incorporation on $\mathrm{Zn}$ sites in the form of electrically active $A s_{Z n}-2 V_{Z n}$ complexes [13-14]. If this hypothesis were correct, the intentional introduction of such a particular defect would constitute a novel approach to semiconductor doping and present a challenging task for defect engineering.

In the case of GaN, the doping with As has been studied with respect to its luminescence behaviour [15-22] and, at higher concentrations, with regards to the formation of $\mathrm{GaAs}_{x} \mathrm{~N}_{1-x}$ alloys and the related modification of the GaN band gap [17,21,23-26]. 
Unfortunately the growth of $\mathrm{GaAs}_{x} \mathrm{~N}_{1-x}$ compounds encounters significant difficulties, one of the reasons being that the most stable polytype of $\mathrm{GaN}$ is hexagonal wurtzite while GaAs crystallizes in the cubic zinc blende structure. However, while, on the As-rich side of the phase diagram, it is possible to incorporate up to $\sim 10-15 \%$ of $N$ into cubic GaAs $[27,28]$, on the N-rich side not more than $\sim 1 \%$ of As in GaN have been achieved [23-26]. In the intermediate region usually the coexistence of hexagonal N-rich GaAsN and cubic As-rich GaNAs phases is observed. As a matter of fact, arsenic in $\mathrm{GaN}$ has been suggested to act as an amphoteric impurity, with its lattice site preference depending on the doping character of the material, i.e. mostly substitutional Ga in $p$-type but also substitutional $\mathrm{N}$ in $n$-type [29,30].

In order to understand the properties of the heavy group $\mathrm{V}$ impurities in $\mathrm{ZnO}$ and $\mathrm{GaN}$ it is hence essential to assess whether they prefer substitutional cation or anion sites. However, so far only few experimental methods have been able to provide reliable knowledge on the lattice sites of these elements in $\mathrm{ZnO}$ and GaN. Desnica et al [31,32] concluded from Mössbauer effect studies that ${ }^{119} \mathrm{Sb}$ implanted into GaN is incorporated in substitutional Ga sites. Since their finding was in contrast to all common III-V compounds, where Sb group V foreign atoms occupy the anion site, they termed $\mathrm{Sb}_{\mathrm{Ga}}$ an "antisite" defect, in analogy to $\mathrm{As}_{\mathrm{Ga}}$ antisites, a native defect commonly observed at low concentrations in GaAs crystals. We summarize here recent results on lattice location experiments of radioactive ${ }^{73} \mathrm{As}$ in $\mathrm{ZnO}[33,34]$ and $\mathrm{GaN}$ [35] by means of the emission channeling technique [36], giving direct evidence that As prefers Zn sites in $\mathrm{ZnO}$ and acts as an amphoteric impurity in GaN.

\section{EXPERIMENT}

A typical emission channeling experiment is schematically illustrated in figure 1 . The method uses radioactive probe atoms that have been implanted into a single crystal sample and is based on the fact that charged particles from the nuclear decay (in our case conversion electrons) experience channeling or blocking effects along major crystallographic axes and planes. The resulting anisotropic emission yield from the crystal surface characterizes the lattice site occupied by the probe atoms during decay.

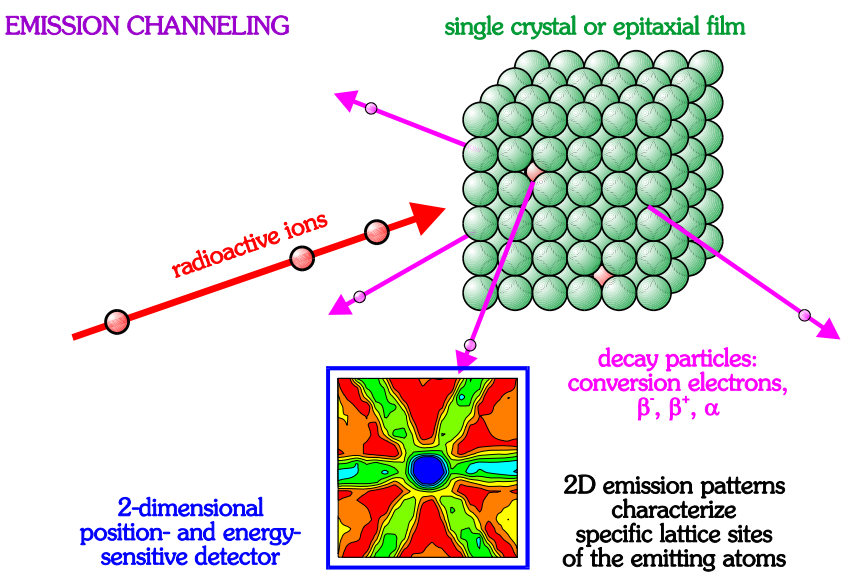

Figure 1. Illustration of a typical emission channeling experiment using radioactive probe atoms implanted into a sample. Note that the size of the single crystal is of course greatly exaggerated in comparison to the detector. Also, many atomic layers contribute to the channeling effect. 
For the experiments described below, single crystalline thin films (thickness $\sim 1 \mu \mathrm{m}$ ) of GaN grown on sapphire and a [0001] oriented $\mathrm{ZnO}$ single crystal were implanted at room temperature with ${ }^{73} \mathrm{As}\left(t_{1 / 2}=80.3 \mathrm{~d}\right)$ at $60 \mathrm{keV}$ energy with fluences between $0.8-7.0 \times 10^{13} \mathrm{~cm}^{-2}$. The production of ${ }^{73} \mathrm{As}$ and the implantation were performed at CERN's on-line isotope separator facility ISOLDE. The As depth profile corresponding to these implantation conditions is centered at a depth of $232 \AA$, with a straggling of $100 \AA$ and the As peak concentration amounts to $0.3-2.7 \times 10^{18}$ atoms $\mathrm{cm}^{-3}$. The maximum As concentration in our experiments was hence still in the $30 \mathrm{ppm}$ regime. The angular emission patterns of the $42.3 \mathrm{keV}$ and $52.1 \mathrm{keV}$ conversion electrons from the ${ }^{73} \mathrm{As} \rightarrow{ }^{73} \mathrm{Ge}$ decay were recorded around the [0001], [1102], [1101] and [2113] directions by means of a $3 \times 3 \mathrm{~cm}^{2}$ sized $22 \times 22$ pixel position-sensitive Si detector $[37,38$ ] at a distance of $30 \mathrm{~cm}$ from the sample. These measurements were carried out at room temperature in the as-implanted state and following $10 \mathrm{~min}$ in situ vacuum $\left(<10^{-5} \mathrm{mbar}\right)$ annealing steps at $300^{\circ} \mathrm{C}, 600^{\circ} \mathrm{C}$ and $900^{\circ} \mathrm{C}$.

The evaluation of the probe atom lattice location was performed by quantitatively comparing the experimental patterns with theoretical ones for different lattice sites, using the two-dimensional fit procedure outlined in Ref. [37]. In the fit procedure, we considered theoretical patterns resulting from emitter atoms at substitutional cation (i.e. $\mathrm{S}_{\mathrm{Zn}}$ and $\mathrm{S}_{\mathrm{Ga}}$ ) and anion sites (i.e. $S_{O}$ and $S_{N}$ ) with varying root mean square (rms) displacements, the main interstitial sites (figure 2, see also Refs. [33,39]), and a diversity of interstitial sites resulting from displacements along or off the $c$-axis. The theoretical emission channeling patterns were calculated by means of the "manybeam" theory of electron diffraction in single-crystals [36]. Details with respect to the structural properties of $\mathrm{GaN}$ and $\mathrm{ZnO}$ used in the simulations have been given previously [39-41].

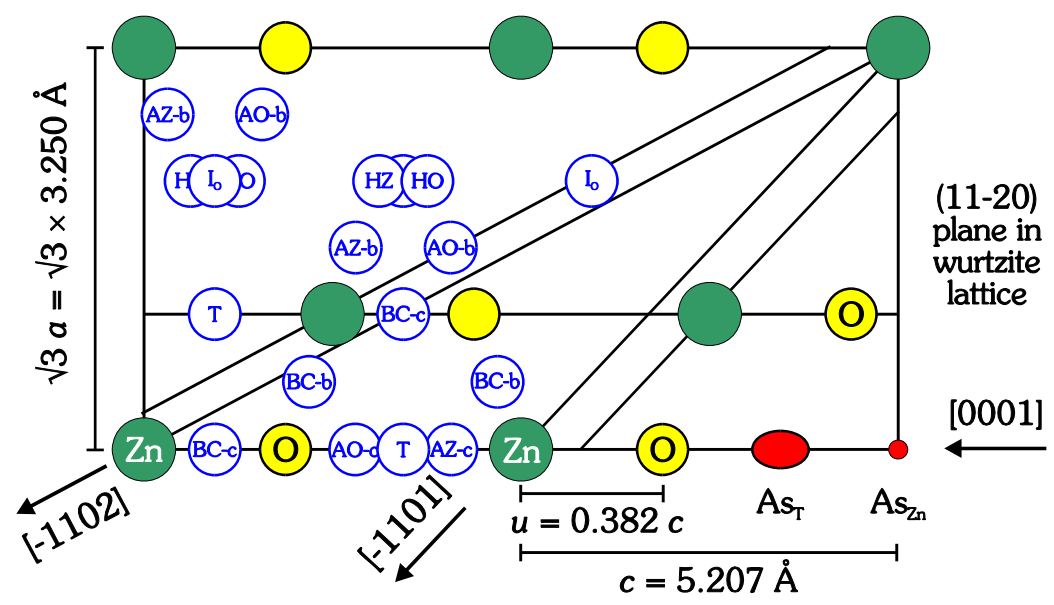

Figure 2. The (1120) plane in the $\mathrm{ZnO}$ wurtzite lattice, showing the location of substitutional $\mathrm{Zn}$ sites, substitutional O sites, bond center BC, antibonding Zn (AZ) and antibonding O (AO), interstitial "octahedral" ( $\left.\mathrm{I}_{\mathrm{O}}\right)$, "tetrahedral" $(\mathrm{T})$ and "hexagonal" (HZ and $\left.\mathrm{HO}\right)$. "-c" denotes sites within and "-b" basal to the $c$-axis. The small red circle and larger ellipse in the lower right hand corner indicate the estimated experimental error in determining the location of ${ }^{73}$ As atoms on substitutional $\mathrm{Zn}$ and interstitial T sites from the emission channeling experiments.

From Ref. [33]. ㄷ The American Physical Society 2005. 


\section{RESULTS}

\section{Lattice location of As in ZnO}

Figure 3 shows typical angular distribution patterns of conversion electrons in the vicinity of the four major crystalline directions of the $\mathrm{ZnO}$ sample. The patterns were measured at room temperature after $600^{\circ} \mathrm{C}$ annealing but are in fact very similar to what is observed already in the as-implanted state (shown in Ref. [34]) or after $300^{\circ} \mathrm{C}$ annealing (shown in Ref. [33]). The best fit of theoretical patterns to the experimental ones was obtained for $80(7) \%$ of probe atoms at $S_{\mathrm{Zn}}$ sites, and the remainder on "random" sites (random sites are lattice positions which cause only negligible anisotropy in the emission patterns, for instance probe atoms in positions of very low symmetry or in heavily damaged surroundings). The rms displacements from the ideal $\mathrm{Zn}$ positions were in all cases around $0.07-0.17 \AA$, i.e. somewhat larger than the thermal vibration amplitude of the $\mathrm{Zn}$ atoms, $u_{1}(\mathrm{Zn})=0.080 \AA$. Major fractions of emitter atoms on other highsymmetric lattice sites can be ruled out. The theoretical channeling patterns from emitter atoms on substitutional $\mathrm{O}$ sites, for instance, are similar to those resulting from substititutional $\mathrm{N}$ sites in GaN shown below in figure $4(\mathrm{~m}-\mathrm{n})$ and are clearly incompatible with the experimental patterns. Including additional fractions of emitter atoms on $\mathrm{S}_{\mathrm{O}}$ sites (besides $\mathrm{S}_{\mathrm{Zn}}$ and random sites) in the fits, the resulting fractions were smaller than $5 \%$ percent, which is within the error bars of the accuracy of the method.

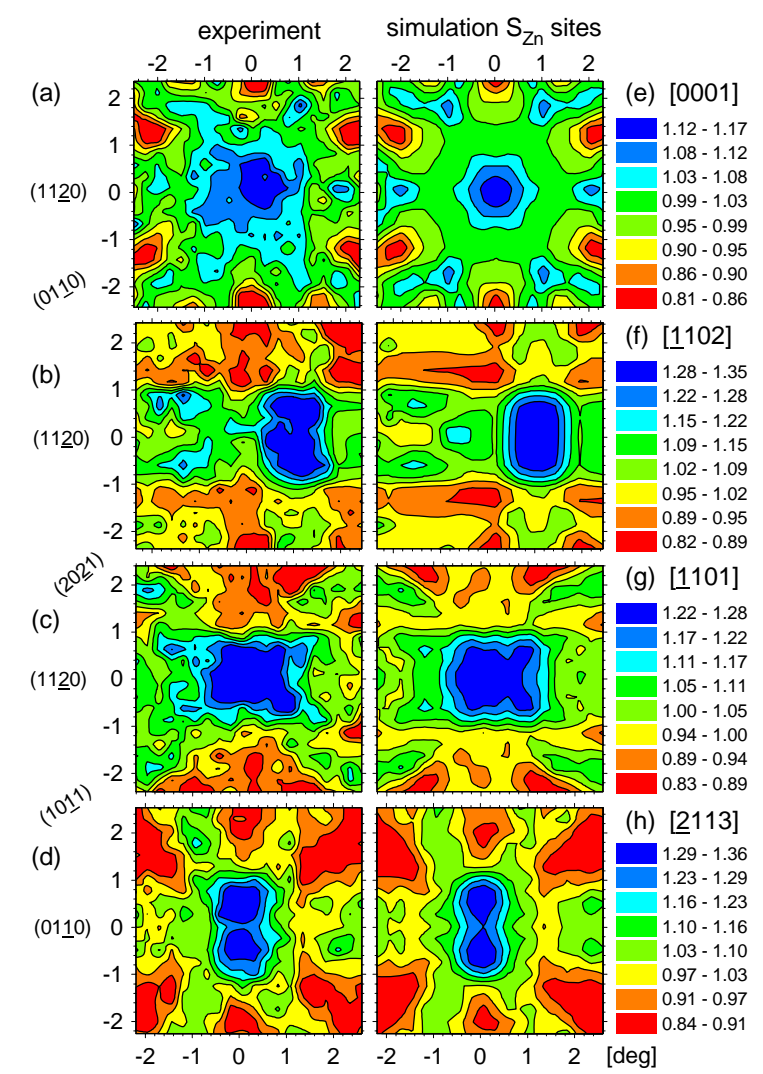

Figure 3. Angular distribution of conversion electrons from ${ }^{73} \mathrm{As}$ in $\mathrm{ZnO}$ following $600^{\circ} \mathrm{C}$ annealing around the [0001] (a), [1102] (b), [1101] (c) and [2113] (d) axis. (e-h): best fits of the channeling patterns for each direction, corresponding to $80(7) \%$ of probe atoms at $\mathrm{S}_{\mathrm{Zn}}$ sites. 
In contrast to all experimental channeling patterns in the as-implanted state and following $300^{\circ} \mathrm{C}$ and $600^{\circ} \mathrm{C}$ vacuum annealing, which could be very well fitted assuming only As on substitutional $\mathrm{Zn}$ sites and on random sites, following $900^{\circ} \mathrm{C}$ vacuum annealing an additional lattice site had to be introduced in the fits. Whereas the [0001] patterns remained relatively unaltered, showing that the probe atoms were still well-aligned with the c-axis, satisfactory fit results for the off-surface directions [1102], [1101] and [2113] could only be obtained when also ${ }^{73}$ As atoms located within $0.4 \AA$ from tetrahedral interstitial $\mathrm{T}$ sites were allowed in the fits [33]. Note that the $\mathrm{T}$ sites are the major interstitial sites in the wurtzite structure that are aligned with the c-axis, making their [0001] emission channeling pattern identical to those of $\mathrm{S}_{\mathrm{Zn}}$ and $\mathrm{S}_{\mathrm{O}}$ sites.

\section{Lattice location of As in GaN}

Following implantation of ${ }^{73} \mathrm{As}$ in $\mathrm{GaN}$, the experimental channeling patterns could not satisfactorily described by As on cation sites only.
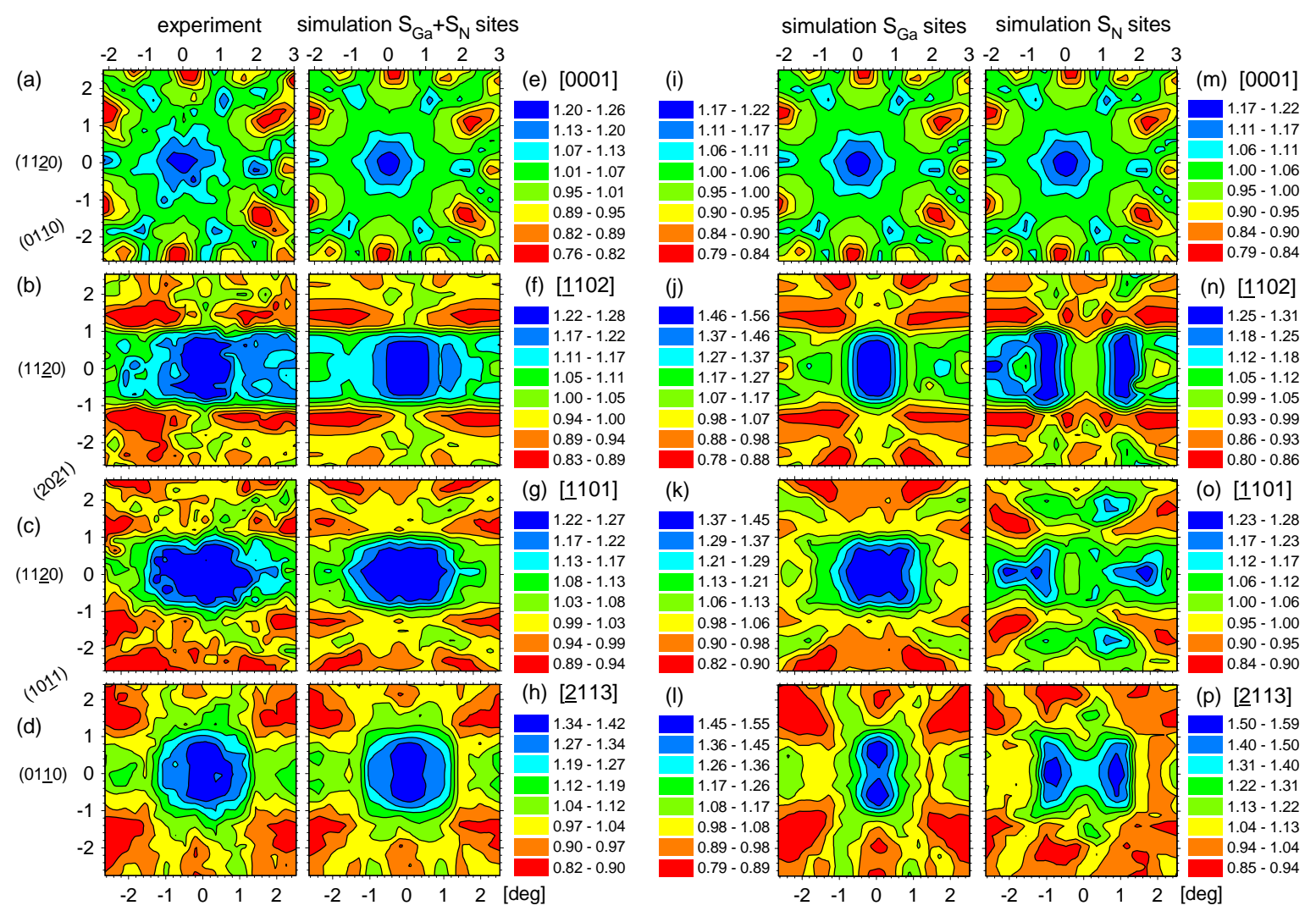

Figure 4. Experimental emission channeling patterns from ${ }^{73} \mathrm{As}$ in $\mathrm{GaN}$ in the as-implanted state around the [0001] (a), [1102] (b), [11101] (c) and [2113] (d) axis. (e-h): best fits of the channeling patterns for each direction, corresponding to $62(5) \%$ of probe atoms at $\mathrm{S}_{\mathrm{Ga}}$ and $45(3) \%$ at $\mathrm{S}_{\mathrm{N}}$ sites. Panels (i-l) are theoretical patterns for $100 \%$ of emitter atoms on $\mathrm{S}_{\mathrm{Ga}}$ sites and panels (m-p) for $100 \%$ on $S_{N}$ sites. Note that the [0001] patterns for $S_{G a}$ and $S_{N}$ are identical since both sites are aligned with the atomic rows along the c-axis. 
As an example we have given in figure 4 (a-d) above the emission channeling patterns measured in the as-implanted state, which are compared to theoretical patterns for $100 \%$ of emitter atoms on substitutional Ga sites [figure 4 (i-l)] and for $100 \%$ on substitutional $\mathrm{N}$ sites [figure $4(\mathrm{~m}-\mathrm{p})$ ]. The comparison of experimental and theoretical patterns shows that the measured emission channeling effects are certainly dominated by emitter atoms on Ga sites, which either produce relatively strong and wide axial effects or a close double peak in case of the [2113] axis. In contrast, emitter atoms on $\mathrm{N}$ sites are characterized by multiple-peak effects which are generally quite far apart and weaker. Obviously, there are certain features in the experimental patterns which cannot be explained by As on Ga sites alone but require the presence of substantial amounts of As on N sites. Most obvious in that respect are the [2113] patterns, where pure Ga sites would result in an intense close double peak within the (1212) plane [vertical in figure $4(\mathrm{l})$ ], while $\mathrm{N}$ sites would be characterized by a pronounced double peak within the (0110) plane [horizontal in figure $4(\mathrm{p})$ ]. What is actually observed in the experiment is a superposition of the $\mathrm{Ga}$ and $\mathrm{N}$ site patterns, with the best fits obtained for 62(5)\% of As on Ga sites and $45(3) \%$ on $\mathrm{N}$ sites. In comparsion to the fit results assuming pure Ga site occupation, the chi square of the [1102], [1101] and fits improved by 39\%, 29\% and 65\% upon introducing the fraction of emitter atoms on substitutional $\mathrm{N}$ sites. The rms displacements of As from the ideal $\mathrm{S}_{\mathrm{Ga}}$ and $\mathrm{S}_{\mathrm{N}}$ sites could not be independently derived in an unambiguous way from the fit results, however, both were in the range $\sim 0.08-0.19 \AA$, somewhat larger than the thermal vibration amplitudes of $\mathrm{Ga}(0.074 \AA)$ and $\mathrm{N}(0.081 \AA)$ and corresponding to $4-10 \%$ of the bond length of $b=1.937 \AA$ in GaN.

\section{Comparison of $\mathrm{ZnO}$ and $\mathrm{GaN}$ as a function of annealing temperature}

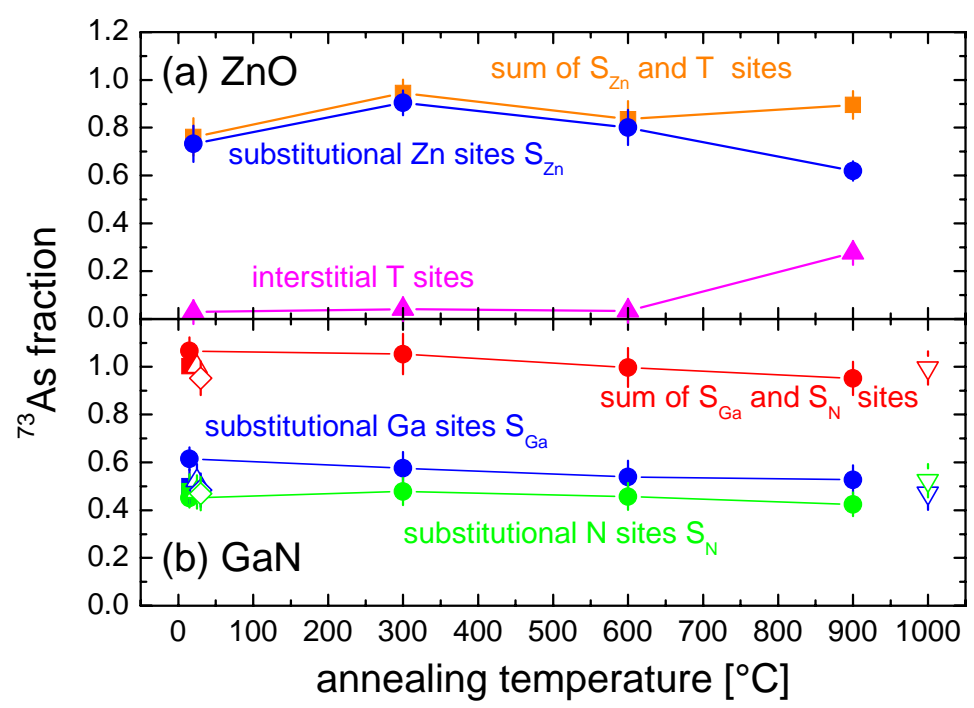

Figure 5. Fitted fractions of ${ }^{73} \mathrm{As}$ probe atoms on the considered lattice sites in $\mathrm{ZnO}$ (a) and GaN (b) as a function of annealing temperature. The four different symbols (circles, squares, triangles and diamonds) in panel (b) correspond to four different GaN crystals investigated, of which only one was subjected to a full annealing sequence, while the other three were only studied in the asimplanted state or following $1000^{\circ} \mathrm{C}$ treatment. All annealings were for $10 \mathrm{~min}$ under vacuum except for the $\mathrm{GaN}$ sample annealed at $1000^{\circ} \mathrm{C}$, which was under $\mathrm{N}_{2}$ atmosphere for $30 \mathrm{~min}$. 
Figure 5 above summarizes and compares the lattice location results for ${ }^{73} \mathrm{As}$ in $\mathrm{ZnO}$ and $\mathrm{GaN}$ as a function of annealing temperature. In both cases quite high fractions of probe atoms occupied regular, high-symmetric (i.e. non-random) lattice sites already in the as-implanted state. Correspondingly, major annealing steps, which would be characterized by a further increase in these fractions, were absent. Apart from the partial site change from $\mathrm{S}_{\mathrm{Zn}}$ to interstitial $\mathrm{T}$ sites, which occurred during $900^{\circ} \mathrm{C}$ annealing in $\mathrm{ZnO}$, the fractions were quite stable. In one $\mathrm{GaN}$ sample the sum of all fitted fractions is somewhat higher than $100 \%$. This is due to an experimental uncertainty which is introduced when correcting for the background of scattered electrons ( $\pm 1 / 10$ of each fraction), and affects all fractions in the same way. The fraction of As on $\mathrm{N}$ sites appears to be somewhat higher in the $\mathrm{GaN}$ sample which was subjected to $1000^{\circ} \mathrm{C}$ annealing, which might indicate that high-temperature treatment can influence to some extent the preferred lattice site of As. However, the difference is relatively small and more experiments would be needed in order to confirm whether this is a significant effect.

\section{DISCUSSION}

Based on the results of their Mössbauer effect studies, which suggested the incorporation of implanted Sb on Ga sites in GaN, Desnica et al proposed that, besides chemical similarities, the atomic or ionic size of an impurity can play a decisive role in determining its preferred lattice site [31,32]. Similar arguments were put forward by Pearton et al [1] regarding the incorporation of the heavy group $\mathrm{V}$ elements $\mathrm{P}$, As and $\mathrm{Sb}$ in $\mathrm{ZnO}$. It was argued that the large ionic size of these impurities in their 3- charge state would prohibit their incorporation on substitutional $\mathrm{O}$ sites, while the much smaller ionic size of the $3+$ charge state would favor replacing the $\mathrm{Zn}^{2+}$ ions. In table I we compare the various relevant ionic and covalent radii of the elements under consideration, giving in addition their Pauling electronegativity, which can be used as a rough criterion for chemical similarity. As is obvious from these data, the substitution of an $\mathrm{O}^{2-}$ $(1.38 \AA)$ ion by $\mathrm{As}^{3-}(2.22 \AA)$ would heavily disturb the $\mathrm{ZnO}$ lattice. On the other hand, the much smaller size of $\mathrm{As}^{3+}(0.58 \AA)$ provides an almost perfect replacement for $\mathrm{Zn}^{2+}$ ions $(0.60 \AA)$ ). This is schematically illustrated in figure 6 . In addition, the difference in the electronegativity of As and $\mathrm{Zn} \mathrm{(0.4)} \mathrm{is} \mathrm{smaller} \mathrm{than} \mathrm{the} \mathrm{difference} \mathrm{between} \mathrm{As} \mathrm{and} \mathrm{O} \mathrm{(1.5),} \mathrm{so} \mathrm{that} \mathrm{in} \mathrm{this} \mathrm{case} \mathrm{our}$ experimental findings regarding the preference of As for $\mathrm{Zn}$ sites can be well explained on the basis of such relatively simple considerations. The preference of As and Sb for Zn sites has also been proposed by Limpijungnong et al $[13,14]$ based on results of first-principles calculations using the local density approximation. These authors suggested in addition that the experimentally observed p-type character of As- or Sb-doped $\mathrm{ZnO}$ results from the formation of $A s_{\mathrm{Zn}}-2 V_{\mathrm{Zn}}$ or $\mathrm{Sb}_{\mathrm{Zn}}-2 V_{\mathrm{Zn}}$ complexes, which act as structural acceptors, in contrast to the simple "chemical” acceptors $\mathrm{As}_{\mathrm{O}}$ or $\mathrm{Sb}_{\mathrm{O}}$. The implications of our experimental observations with respect to distinguishing between these two models is discussed in a separate section below. 
Table I. Pauling electronegativity, covalent radius, relevant charge states and corresponding ionic radii for the host elements Zn, Ga, N, O and the group V impurities P, As and Sb. The ionic radii are from: ${ }^{1}$ Ref. [42] for coordination number IV, ${ }^{2}$ Ref. [42] for coordination number VI, ${ }^{3}$ Ref. [43] for coordination number VI

\begin{tabular}{|l|l|l|l|l|}
\hline Element & $\begin{array}{l}\text { Pauling electro- } \\
\text { negativity }\end{array}$ & $\begin{array}{l}\text { covalent } \\
\text { radius }[\AA]\end{array}$ & $\begin{array}{l}\text { charge } \\
\text { state }\end{array}$ & ionic radius $[\AA]$ \\
\hline Zn & 1.6 & 1.25 & $2+$ & $0.60^{1}$ \\
\hline $\mathrm{O}$ & 3.5 & 0.73 & $2-$ & $1.38^{1}$ \\
\hline $\mathrm{Ga}$ & 1.6 & 1.26 & $3+$ & $0.47^{1}$ \\
\hline $\mathrm{N}$ & 3.0 & 0.75 & $3-$ & $1.46^{1}$ \\
\hline $\mathrm{P}$ & 2.1 & 1.06 & $3-$ & $2.12^{3}$ \\
& & & $3+$ & $0.44^{2}$ \\
& & & $5+$ & $0.17^{1}$ \\
\hline As & 2.0 & 1.20 & $3-$ & $2.22^{3}$ \\
& & & $3+$ & $0.58^{2}$ \\
& & & $5+$ & $0.335^{1}$ \\
\hline Sb & 1.9 & 1.40 & $3-$ & $2.45^{3}$ \\
& & & $3+$ & $0.74^{1}$ \\
& & & $5+$ & $0.60^{2}$ \\
\hline
\end{tabular}
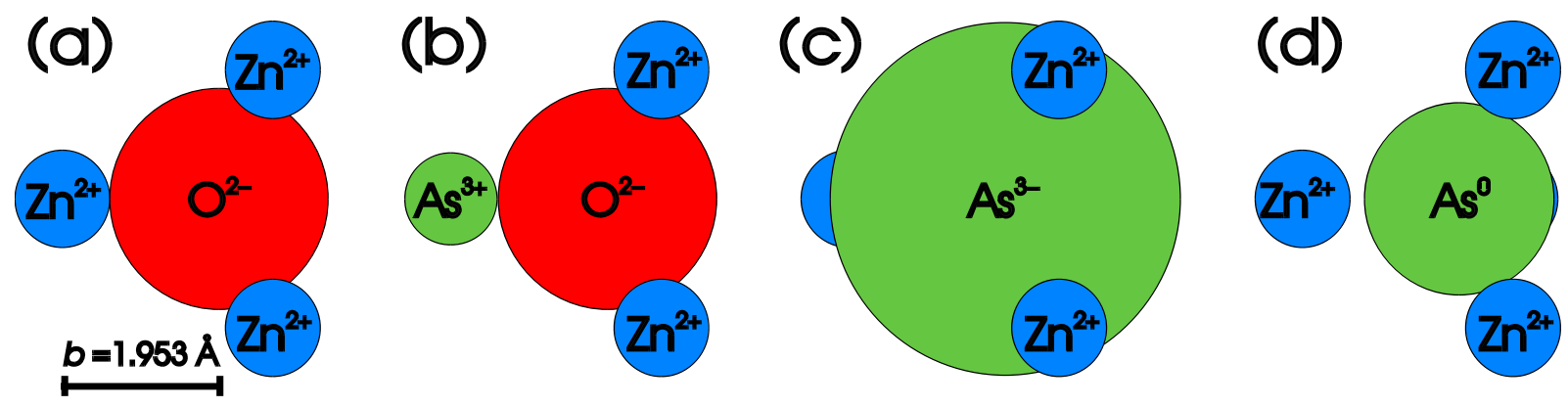

Figure 6. Schematics illustrating the ionic sizes of $\mathrm{Zn}, \mathrm{O}$ and $\mathrm{As}$ in zinc oxide. Note that the structure has been rotated in such a way that the straight line connecting the central $\mathrm{O}$ or As atom with the leftmost atom lies within the horizontal plane. (a): In the undisturbed $\mathrm{ZnO}$ lattice, the bond length $b=1.953 \AA$ between neighboring $\mathrm{Zn}$ and $\mathrm{O}$ atoms almost exactly matches the sum of the ionic radii of $\mathrm{Zn}^{2+}(0.60 \AA)$ and $\mathrm{O}^{2-}(1.38 \AA)$, illustrating the comparatively strong ionic character of the bonds. (b): The leftmost $\mathrm{Zn}^{2+}$ ion has been replaced with an $\mathrm{As}^{3+}$ ion of radius of

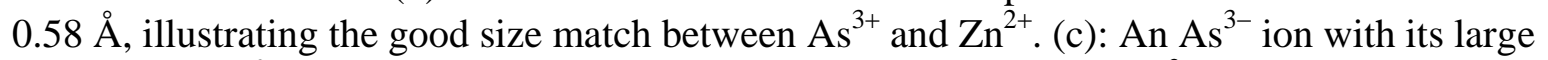
radius of $2.22 \AA$ does not fit within the cage of the surrounding four $\mathrm{Zn}^{2+}$ ions. (d): In contrast, a neutral $\mathrm{As}^{0}$ atom with its covalent radius of $1.20 \AA$ would fit within the cage of the surrounding four $\mathrm{Zn}^{2+}$ ions.

The case of As in GaN is somewhat different. In GaN, the ionic radii of $\mathrm{As}^{3+}$ and $\mathrm{Ga}^{3+}$ are also well matched ( $0.58 \AA$ vs $0.62 \AA$ ), while the size-mismatch between the $\mathrm{As}^{3-}$ and $\mathrm{N}^{3-}$ anions is considerably smaller (2.22 $\AA$ vs $1.71 \AA$ ) than in $\mathrm{ZnO}$ but still significant. The electronegativity 
difference of As and $\mathrm{N}(1.0)$ is also smaller than in $\mathrm{ZnO}$ but still larger than the difference between As and Ga (0.4). However, despite of these simple considerations, which would also suggest that As replaces the Ga cations, apparently the chemical similarity between As and $\mathrm{N}$ is strong enough in order to allow for roughly half of it to be incorporated on $\mathrm{N}$ sites. The amphoteric character of As in GaN has been predicted by Van De Walle and Neugebauer [29] and Ramos et al [30] from first-principles calculations, and our experimental results confirm their theoretical findings.

\section{Relevance of current results with respect to the acceptor model of As in $\mathrm{ZnO}$}

Unfortunately our experiments cannot settle the interesting issue whether substitutional As on oxygen sites $\left(\mathrm{As}_{\mathrm{O}}\right)$, as is often assumed $[3,4,8]$, or $\mathrm{As}_{\mathrm{Zn}}-2 V_{\mathrm{Zn}}$ complexes, as was suggested by Limpijumnong et al $[13,14]$, are responsible for the acceptor action in As-doped $\mathrm{ZnO}$. The fact that implanted As prefers the substitutional Zn sites is clearly a strong argument in favor of the $A s_{Z n}-2 V_{Z n}$ model. However, since the channeling effect is not suitable to characterize the immediate neighbourhood of the probe atoms and we hence cannot probe whether the substitutitional $\mathrm{As}_{\mathrm{Zn}}$ is paired with one or more vacancies, we cannot fully confirm the structural $A s_{Z n}-2 V_{Z n}$ model. What we find is that the rms displacements of the probe atoms from the ideal substitutional $\mathrm{Zn}$ sites are relatively small, thus excluding the possibility of large lattice relaxations other than breathing mode relaxations. Our results also showed that following vacuum annealing at $900^{\circ} \mathrm{C}$ a significant fraction of As probe atoms (almost 30\%) was found within $0.4 \AA$ from interstitial T sites. It is known that annealing $\mathrm{ZnO}$ above $500^{\circ} \mathrm{C}$ under O-poor conditions can result in $\mathrm{O}$ loss and thus change the stoichiometry in the near-surface region [1]. Given the relatively shallow depth profile of the implanted As, part of the implanted probe atoms may therefore have reacted for instance with $\mathrm{O}$ vacancies upon annealing at $900^{\circ} \mathrm{C}$, forming a complex. However, this complex is probably not the $\mathrm{As}_{\mathrm{Zn}}-2 V_{\mathrm{Zn}}$ proposed by Limpijumnong et al, for which they predicted an As position halfway from the ideal $\mathrm{Zn}$ position towards the antibonding $\mathrm{Zn}$ site (AZ-c in figure 2), i.e. 0.4-0.5 $\AA$ from $\mathrm{S}_{\mathrm{Zn}}$, since our oxygen-poor annealing conditions should not favour its formation and in addition we found the As within $0.4 \AA$ from interstitial T sites, i.e. $\sim 1.6 \AA$ from $\mathrm{S}_{\mathrm{Zn}}$.

On the other hand, while we have proven that the vast majority of As prefers occupying $\mathrm{Zn}$ sites, which is certainly not an encouraging perspective for As as a chemical dopant, we do not have any information with respect to its electrical activity in our samples. This issue is of particular relevance, since it has been reported that not more than $5-13 \%$ of incorporated As can be electrically activated in $\mathrm{ZnO}$ [4,5,7]. The maximum concentration of As in our implanted sample (around $7.6 \times 10^{18} \mathrm{~cm}^{-3}$ ) is comparable with the As concentration used in most other studies. Although we are sure that in our case less than $5 \%$ of the implanted As occupied $O$ sites, it cannot be excluded that by means of using different doping techniques, e.g. doping during molecular beam epitaxy (MBE) growth, around 10\% of As might be incorporated on O sites and thus be responsible for the $p$-type conductivity reported in the literature. The amphoteric character of As in GaN actually provides an additional argument in favour of $\mathrm{As}_{\mathrm{O}}$ acceptors, since obviously the size-mismatch on anion sites, which is less pronounced in GaN but still present, is not able to prevent As from occupying anion positions in GaN. We may therefore conclude that the question of the nature of the As acceptor in $\mathrm{ZnO}$ is certainly still open for discussion. 


\section{Relevance of current results with respect to miscibility gap in GaAsN compounds}

As was already mentioned in the introduction, it has not been possible so far to grow single phase $\mathrm{GaAs}_{x} \mathrm{~N}_{1-x}$ with $x$ values in the range $0.1<\mathrm{x}<0.99$, which is known as the so-called "miscibility gap" for this type of ternary compound. The amphoteric character of As in GaN and the fact that $\mathrm{As}_{\mathrm{Ga}}$ "anti-sites" are not at all minority defects provide additional aspects to be taken into account for an explanantion of this "miscibility gap". It is quite obvious that As atoms on Ga sites do not contribute to the stability of the compound, since no chemical bonds will be formed with the neighboring $\mathrm{N}$ or As atoms. Our findings clearly confirm the theoretical predictions of Van De Walle and Neugebauer [29] who have pointed out $\mathrm{As}_{\mathrm{Ga}}$ anti-site formation as a problem with respect to the growth of GaAsN compounds, while a large number of theoretical studies have completely neglected this possibility [44-55]. While the preservation of chemical bonds probably holds in the case of GaAs lightly doped with N, trying to explain the properties of GaNrich alloys doped with As based on the assumption that all chemical bonds between $\mathrm{Ga}$ and $\mathrm{N}$ or $\mathrm{Ga}$ and As are preserved will definitely yield a too simplified picture. It also seems doubtful whether the properties of GaAsN alloys, e.g. their band structure and pronounced bandgap bowing, or their luminescence properties can be fully described without taking into account the formation of $\mathrm{As}_{\mathrm{Ga}}$ defects.

\section{CONCLUSIONS}

We have determined the lattice location of implanted As in $\mathrm{ZnO}$ and $\mathrm{GaN}$ by means of conversion electron emission channeling from radioactive ${ }^{73} \mathrm{As}$. In contrast to what one might expect from its nature as a group $\mathrm{V}$ element, we find that As does not occupy substitutional $\mathrm{O}$ sites in $\mathrm{ZnO}$ but in its large majority substitutional $\mathrm{Zn}$ sites. Arsenic in $\mathrm{ZnO}$ is thus an interesting example for an impurity in a semiconductor where the major impurity lattice site is determined by atomic size and electronegativity rather than its position in the periodic system. The preference for cation or anion sites is different in the case of As implanted into GaN, where we found roughly half of the implanted As atoms occupying $\mathrm{Ga}$ and the other half $\mathrm{N}$ sites. The amphoteric character of As therefore certainly plays a role in explaining the extreme difficulties in growing high quality $\mathrm{GaAs}_{x} \mathrm{~N}_{1-x}$ alloys with values of $x$ above a few percent.

\section{ACKNOWLEDGMENTS}

This work was funded by the Portuguese Foundation for Science and Technology (FCT, project POCI-FP-63911-2005) and by the European Commission (EURONS project RII3-CT2004-506065). E. Rita and A.C. Marques acknowledge financial support from FCT, Portugal. 


\section{REFERENCES}

1. S.J. Pearton, D.P. Norton, K. Ip, Y.W. Heo, and T. Steiner, J. Vac. Sci. Tech. B 22, 932 (2004), and references therein.

2. D.C. Look, Semicond. Sci. Technol. 20, S55 (2005), and references therein.

3. Y.R. Ryu, S. Zhu, D.C. Look, J.M. Wrobel, H.M. Jeong, and H.W. White, J. Cryst. Growth 216, 330 (2000).

4. Y.R. Ryu, T.S. Lee, and H.W. White, Appl. Phys. Lett. 83, 87 (2003).

5. D.C. Look, G.M. Renlund, R.H. Burgener, and J.R. Sizelove, Appl. Phys. Lett. 85, 5269 (2004).

6. V. Vaithianathan, B. Lee, and S.S. Kim, Appl. Phys. Lett. 86, 062101 (2005).

7. G. Braunstein, A. Muraviev, H. Saxena, N. Dhere, V. Richter, and R. Kalish, Appl. Phys. Lett. 87, 192103 (2005).

8. V. Vaithianathan, B.T. Lee, C.H. Chang, K. Asokan, and S.S. Kim, Appl. Phys. Lett. 88, 112103 (2006).

9. H.S. Kang, G.H. Kim, D.L. Kim, H.W. Chang, B.D. Ahn, and S.Y. Lee, Appl. Phys. Lett. 89, 181103 (2006).

10. T. Aoki, Y. Shimizu, A. Miyake, A. Nakamura, Y. Nakanishi, and Y. Hatanaka, phys. stat. solidi (b) 229, 911 (2002).

11. F.X. Xiu, Z. Yang, L.J. Mandalapu, D.T. Zhao, J.L. Liu, and W.P. Beyermann, Appl. Phys. Lett. 87, 152101 (2005).

12. O. Lopatiuk-Tirpak, L. Chernyak, F.X. Xiu, J.L. Liu, S. Jang, F. Ren, S.J. Pearton, K. Gartsman, Y. Feldmann, A. Osinsky, and P. Chow, J. Appl. Phys. 100, 086101 (2006).

13. S. Limpijumnong, S.B. Zhang, S.H. Wei, and C.H. Park, Phys. Rev. Lett. 92, 155504 (2004).

14. S. Limpijumnong, M.F. Smith, and S.B. Zhang, Appl. Phys. Lett. 89, 222113 (2006).

15. J.I. Pankove and J.A. Hutchby, J. Appl. Phys. 47, 5376 (1976).

16. R.D. Metcalfe, D. Wickenden, and W.C. Clark, J. Lumin. 16, 405 (1978).

17. A.J. Winser, S.V. Novikov, C.S. Davis, T.S. Cheng, C.T. Foxon, and I. Harrison, Appl. Phys. Lett. 77, 2506 (2000).

18. B. Gil, A. Morel, T. Taliercio, P. Lefebvre, C.T. Foxon, I. Harrison, A.J.Winser, and S.V. Novikov, Appl. Phys. Lett. 79, 69 (2001).

19. A. Bell, F.A. Ponce, S.V. Novikov, C.T. Foxon, and I. Harrison, Appl. Phys. Lett. 79, 3239 (2001).

20. H.Y. Huang, J.Q. Xiao, C.S. Ku, H.M. Chung, W.K. Chen, W.H. Chen, M.C. Lee, and H.Y. Lee, J. Appl. Phys. 92, 4129 (2002).

21. C.T. Foxon, I. Harrison, S.V. Novikov, A.J. Winser, R.P. Campion, and T. Li, J. Phys.: Condens. Matter 14, 3383 (2002).

22. A. Stötzler, R. Weissenborn, M. Deicher, and the ISOLDE collaboration, MRS Internet J. Nitride Semicond. Research 5S1, W12.9 (2000), see also Mater Res. Soc. Symp. Proc. 595, W12.9 (2000).

23. X. Li, E.E. Reuter, S.G. Bishop, and J.J. Coleman, Appl. Phys. Lett. 72, 1990 (1998).

24. R. Kuroiwa, H. Asahi, K. Asami, S.J. Kim, K. Iwata, and S. Gonda, Appl. Phys. Lett. 73, 2630 (1998).

25. Y. Zhao, F. Deng, S.S. Lau, and C.W. Tu, J. Vac. Sci. Technol. B 16, 1297 (1998).

26. H. Na, H.J. Kim, E. Yoon, C. Sone, and Y. Park, J. Cryst. Growth 248, 437 (2003). 
27. W.G. Bi and C.W. Tu, Appl. Phys. Lett. 70, 1608 (1997).

28. Y. Qiu, S.A. Nikishin, H. Temkin, N.N. Faleev, and Y.A. Kudriavtsev, Appl. Phys. Lett. 70, 3242 (1997).

29. C.G. Van De Walle and J. Neugebauer, Appl. Phys. Lett. 76, 1009 (2000).

30. L.E. Ramos, J. Furthmüller, J.R. Leite, L.M.R. Scolfaro, and F Bechstedt, Phys. Rev. B 68, 085209 (2003).

31. U.V. Desnica, N. Ravi, H. Andreasen, and H. De Waard, Solid State Comm. 60, 59 (1986).

32. U.V. Desnica, Solid State Comm. 69, 411 (1989).

33. U. Wahl, E. Rita, J.G. Correia, A.C. Marques, E. Alves, J.C. Soares, and the ISOLDE collaboration, Phys. Rev. Lett. 95, 215503 (2005).

34. U. Wahl, E. Rita, J.G. Correia, A.C. Marques, E. Alves, J.C. Soares, and the ISOLDE collaboration, accepted for publication in Superlattices and Microstructures.

35. U. Wahl, J.G. Correia, J.P. Araújo, E. Rita, J.C. Soares, and the ISOLDE collaboration, submitted to Appl. Phys. Lett.

36. H. Hofsäss and G. Lindner, Phys. Rep. 210, 121 (1991).

37. U. Wahl, J.G. Correia, A. Czermak, S.G. Jahn, P. Jalocha, J.G. Marques, A. Rudge, F. Schopper, J.C. Soares, A. Vantomme, P. Weilhammer, and the ISOLDE collaboration, Nucl. Instr. Meth. A 524, 245 (2004).

38. A.C. Marques, U. Wahl, J.G. Correia, M.R. Silva, A. Rudge, P. Weilhammer, J.C. Soares, and the ISOLDE collaboration, Nucl. Instr. Meth. A 572, 1056 (2007).

39. U. Wahl, A. Vantomme, G. Langouche, J.P. Araújo, L. Peralta, J.G. Correia, and the ISOLDE collaboration, J. Appl Phys. 88, 1319 (2000).

40. U. Wahl, E. Rita, J.G. Correia, E. Alves, J.P. Araújo, and the ISOLDE collaboration, Appl. Phys. Lett. 82, 1173 (2003).

41. U. Wahl, E. Rita, J.G. Correia, E. Alves, J.C. Soares, and the ISOLDE collaboration, Phys. Rev. B 69, 012102 (2004).

42. R.D. Shannon, Acta Cryst. A 32, 751 (1976).

43. The CRC Handbook of Chemistry and Physics, $50^{\text {th }}$ edition, ed. R.C. Weast (CRC Press, Boca Raton 1970) p. F-152.

44. L. Bellaiche, S.H. Wei, and A. Zunger, Phys. Rev. B 54, 17568 (1996).

45. L. Bellaiche, S.H. Wei, and A. Zunger, Appl. Phys. Lett. 70, 3558 (1997).

46. L. Bellaiche, S.H. Wei, and A. Zunger, Phys. Rev. B 56, 10233 (1997).

47. T. Mattila and A. Zunger, Phys. Rev. B 58, 1367 (1998).

48. A. Zunger, phys. stat. sol. (b) 216, 117 (1999).

49. T. Mattila and A. Zunger, Phys. Rev. B 59, 9943 (1999).

50. C. Göbel, K. Petzke, C. Schrepel, and U. Scherz, Physica B 273, 759 (1999).

51. P.R.C. Kent and A. Zunger, Phys. Rev. B 64, 115208 (2001).

52. L.W. Wang, Phys. Rev. Lett. 88, 256402 (2002).

53. J. Li and L.W. Wang, Phys. Rev. B 67, 033102 (2003).

54. A Jenichen and C. Engler, phys. stat. sol. (b) 241, 1883 (2004).

55. N. Tit, J. Phys D: Appl. Phys. 39, 2514 (2006) 